\title{
Una revisión de la Cadena Datos-Información- Conocimiento desde el Pragmatismo de Peirce
}

\author{
Miguel Ángel GUILLÉn NAVARRO \\ mguillen@ucam.edu \\ Belén LÓPEZ AYUSO \\ bayuso@ucam.edu \\ Escuela Politécnica Superior \\ Departamento de Ciencias Politécnicas \\ Universidad Católica de Murcia \\ Enrique PANIAGUA ARÍs \\ paniagua@um.es \\ José Manuel CADENAS FIGUEREDO \\ jcadenas@um.es \\ Facultad de Informática \\ Departamento de Ingeniería de la Información y las Comunicaciones \\ Universidad de Murcia
}

Recibido: 30/07/2015

Aceptado: 15/10/2015

\section{RESUMEN}

La Cadena Datos-Información-Conocimiento (DIC), denominada "Jerarquía de la Información” o "Pirámide del Conocimiento", es uno de los modelos más importantes en la Gestión de la Información y la Gestión del Conocimiento. Por lo general, la estructuración de la cadena se ha ido definiendo como una arquitectura en la que cada elemento se levanta sobre el elemento inmediatamente inferior; sin embargo no existe un consenso en la definición de los elementos, ni acerca de los procesos que transforman un elemento de un nivel a uno del siguiente nivel. En este artículo se realiza una revisión de la Cadena Datos-Información-Conocimiento examinando las definiciones más relevantes sobre sus elementos y sobre su articulación en la literatura, para sintetizar las acepciones más comunes. Se analizan los elementos de la Cadena DIC desde la semiótica de Peirce; enfoque que nos permite aclarar los significados e identificar las diferencias, las relaciones y los roles que desempeñan en la cadena desde el punto de vista del pragmatismo. Finalmente se propone una definición de la Cadena DIC apoyada en las categorías triádicas de signos y la semiosis ilimitada de Peirce, los niveles de sistemas de signos de Stamper y las metáforas de Zeleny.

Palabras clave: Cadena Datos-Información-Conocimiento, pragmatismo, semiótica, signo, semiosis.

\section{A Review of the Data-Information-Knowledge Chain from the Pragmatism of Peirce}

\begin{abstract}
The Data-Information-Knowledge (DIC) Chain, known as "Information Hierarchy" or "Knowledge Pyramid", is one of the most important models in Information Management and Knowledge Management. In general, the structure of the DIC Chain has been defined as an architecture in which each
\end{abstract}


element stands on the next lower element; however there does not exit a consensus neither about the definition of its elements nor about the processes that transform an item from one level to the next level one. In this paper we review the DIC Chain through the most relevant definitions about its elements and its articulation in the literature, in order to synthesize the most common meanings. In addition, the elements of the DIC Chain are analyzed from the semiotics of Peirce; this approach allows us to clarify the meaning and identify differences, relationships and the roles that they play in the chain from the point of view of pragmatism. Finally we propose a definition of the DIC Chain supported by the Peirce's triadic categories of signs and unlimited semiosis, along with the levels of the Stamper's systems of signs and Zeleny's metaphors.

Keywords: Data-Information-Knowledge Chain, pragmatism, semiotics, sign, semiosis.

\section{Referencia normalizada}

Guillén, M.A.; Paniagua, E.; Cadenas, J.M.; López, B. (2015). "Una revisión de la Cadena DatosInformación-Conocimiento desde el Pragmatismo de Peirce”. Documentación de las Ciencias de la Información, Vol. 38: páginas. 153-177

\section{INTRODUCCIÓN}

Desde que (Bell, 1976) definiera la sociedad post-industrial como aquella que se organiza en torno al conocimiento para lograr el control social y la dirección de la innovación y el cambio; y posteriormente (Drucker, 1993) pusiera al conocimiento en el centro de la producción de la riqueza; surgen, a partir de la década de los noventa del siglo pasado, toda una serie de modelos de lo que se denomina gestión del conocimiento para apoyar la nueva economía basada en el conocimiento.

Según (Davenport and Prusak, 1998, p. 52), “cuando las empresas interactúan con el entorno, absorben información, la convierten en conocimiento, y realizan acciones basadas en ella en combinación con su experiencia, valores y normas internas”; y para (Ros García, 2006, p. 279-280), “toda empresa es un sistema de información” y "la principal ventaja de una organización es saber hacer uso de esta materia prima de forma eficaz"; por tanto, "la información es la materia prima y el producto elaborado es el conocimiento [...] el valor verdadero de la información”. (Malhotra, 1998, p. 59) define la gestión del conocimiento como "el proceso organizacional que busca la combinación sinérgica del tratamiento de los datos y la información mediante las capacidades de las tecnologías de la información y las capacidades innovadoras de las personas", mientras que (Sveiby, 2000, p. 319), la define como "el arte de crear valor con los activos intangibles de una organización”.

En estas definiciones podemos identificar dos polos axiales sobre los que se apoyan, y desplazan, las diferentes interpretaciones: la información como fuente de conocimiento explícito; y las personas como fuente de conocimiento tácito; ambos como activos intangibles. (Polany, 1962, 1966), al intentar analizar el conocimiento humano, hace una distinción entre el conocimiento explícito, aquel conocimiento codificado que se puede transmitir mediante un lenguaje formal y sistemático; y el tácito, aquel de carácter personal que es difícil comunicar o formalizar. Diferenciación que (Nonaka, 1994) orienta hacia la fuente del conocimiento, estableciendo un emparejamiento 
biunívoco entre tácito-humano y explícito-cósico ${ }^{1}$. Complementariamente a la partición $^{2}$ que realiza Nonaka al relacionar el tipo de conocimiento con su fuente, podemos advertir que las diferentes apreciaciones acerca del conocimiento se asocian a su nivel de formalización, codificación o comunicabilidad. Aspectos que, desde nuestro punto de vista, no tienen una relación directa con el tipo de fuente, ni nos aclaran nada acerca de lo que es el conocimiento; tan solo manifiestan la posibilidad de transmitirlo ${ }^{3}$. En todo caso, hay un consenso en erigir el conocimiento sobre los datos, la información y las personas.

Tal como comenta (Codina, 2002, p. 218), existe una confusión habitual en torno al concepto de información; que se entiende como algo aparentemente inmaterial ${ }^{4}$, aunque los objetos y fenómenos que la poseen son totalmente tangibles; y "se utiliza a veces como un macro concepto de "grano grueso» que no discrimina entre cosas tan distintas [...] como dato, información y conocimiento" (p. 219). Como ya hemos visto, aunque estos tres términos se emplean a menudo en relación con la gestión del conocimiento; no existe una clara distinción entre ellos, ni una manera clara de utilizarlos y aplicarlos. Lo que requiere de un análisis que dilucide su significado, despejando las diferencias, y señalando las relaciones y dependencias que se establecen entre ellos.

Para ello, el proceso realizado en este trabajo, lo hemos organizado de la siguiente manera: primero presentamos las definiciones más relevantes sobre los elementos de la Cadena Datos-Información-Conocimiento (DIC) y a continuación sintetizamos las acepciones más comunes; en la siguiente sección analizamos cada uno de los elementos desde la semiótica de Peirce, enfoque que nos permite descubrir el significado, las relaciones y los roles que desempeñan en la cadena desde el punto de vista del pragmatismo. Finalmente realizamos una definición de los elementos de la Cadena DIC desde el pragmatismo.

\section{Definiciones de los elementos de la Cadena DIC}

Según el Diccionario de la Lengua Española de la $\mathrm{RAE}^{5}$, un dato se entiende como el antecedente o testimonio para llegar al conocimiento exacto de algo o deducir las consecuencias legítimas de un hecho; la información es la comunicación o adquisición de conocimientos que permiten ampliar o precisar los que se poseen sobre una

\footnotetext{
${ }^{1}$ De cosa, como un documento, o la información registrada en cualquier soporte.

${ }^{2}$ En subconjuntos disjuntos.

${ }^{3}$ Que tiene mucha relación con el modelo de comunicación de (Shannon and Weaver, 1971).

4 (Prada Madrid, 2008) la denomina insumo invisible.

5 DRAE [en línea]. 22a ed. [ref. de 22 de julio de 2015]. Disponible en Web: http://www.rae.es.
} 
materia determinada; mientras que el conocimiento es el efecto de conocer (del latín cognoscère), es decir, entender por el ejercicio de las facultades intelectuales, la naturaleza, cualidades y relaciones de las cosas.

Según el Dictionary for Library and Information Science 6 , los datos (en plural) son hechos representados de forma que pueden ser comprendidos, interpretados y comunicados; la información es la representación de datos en una forma comprensible a los que se les ha asignado significado respecto a un contexto de uso; mientras que el conocimiento es información que ha sido comprendida y evaluada en base a la experiencia, incorporada (interiorizada) en el entendimiento intelectual del conocedor de un tema. El conocimiento humano, desde la epistemología, incluye las relaciones que se establecen entre el conocedor, lo conocido y el propio proceso de conocer. En estas definiciones de Reitz encontramos una sutil construcción recurrente de los elementos de la Cadena DIC apoyada en el elemento central de la información.

(Zins, 2007) aplica el método Delphi para obtener los enfoques conceptuales más utilizados en relación con los elementos datos, información y conocimiento. En este estudio, los datos se entiendes inicialmente como estímulos sensoriales, discernibles porque se pueden diferenciar de otros (Boisot and Canals, 2004), que recibimos mediante nuestros sentidos, es decir, de carácter empírico y perceptible; también se entienden como símbolos que cuantifican y/o cualifican una invariante de una serie de observaciones y mediciones, que se pueden repetir, de hechos en bruto acerca del mundo, aunque siempre desconectados, sin procesar ni contextualizar. Al integrarlos en un contexto, estos hechos básicos, atómicos e individuales, elementos de verdad, se interpretan como evidencias sobre las que se pueden identificar correlaciones y obtener conclusiones. Un dato es un objeto atómico físico y manipulable, un recurso externo sin estructura inherente o interrelaciones (Hey, 2004).

En el mismo estudio, la información ${ }^{7}$ se entiende como el resultado de procesar e interpretar los datos de manera que nos aporten significado ${ }^{8}$. La información no se pueden separar los datos del contexto; la información identifica, evalúa, organiza, categoriza, agrupa, selecciona, correlaciona, ordena, agrega, interpreta, analiza y genera un significado, de lo que antes simplemente podíamos considerar como datos. Esa organización, su forma y representación, de los datos se apoya en una ontología que establece las relaciones relevantes e invariantes (Boisot and Canals, 2004) respecto a algún campo: el contexto. La información es la agregación de datos para obtener

${ }^{6}$ Reitz, Joan M. (2004). Dictionary for library and information science [en línea]. [ref. de 22 de julio de 2015]. Disponible en Web: http://www.abc-clio.com/ODLIS/odlis_d.aspx.

${ }^{7}$ Cuya etimología es "dar forma”; por tanto, la información es la forma y estructura que se le da a la substancia de los datos mediante algún tipo de procesamiento, que en la mayoría de los casos conlleva implícitamente el reconocimiento de patrones.

${ }^{8}$ Es la reificación de los datos (Hey, 2004), convertidos en signos. 
conclusiones coherentes, y útiles, acerca del mundo; y por tanto, también es un objeto manipulable y un recurso externo (Hey, 2004).

Y finalmente se entiende por conocimiento el entendimiento y comprensión de la información que nos hemos apropiado e interiorizado. Por tanto existe en nuestra mente, pero también existe su representación externa, que ya no es conocimiento sino información. El conocimiento surge de ideas verificadas y validadas por convención; lo que implica cierto consenso. El conocimiento es el resultado de procesar información y hallar ciertos patrones invariantes que generan un cuerpo coherente de juicios acerca del mundo; es decir, es una comprensión razonada acerca de una visión del mundo. El conocimiento es información verdadera (conclusión) que se corresponde (de forma estable) con otra información verdadera (premisas) en un contexto; pero sólo es una afirmación, realmente una insinuación, que puede ser representada de forma imperfecta mediante la información; información que nos permite comunicar parcialmente dicho conocimiento. El conocimiento es incorporado a nuestras creencias, valores, principios, decisiones y acciones de forma constante, modificando y mutando nuestro conocimiento previo; es decir, es interiorización orientada al uso; por lo que tiene un carácter teleológico. El conocimiento tiene dos modos de existencia: el subjetivo es el del individuo, personal y casi siempre mental; mientras que el objetivo, que sería mejor denominarlo colectivo o público, es un objeto cuya representación es entonces información. Además, desde un punto de vista epistemológico, puede ser práctico (habilidades concretas, como saber conducir un coche), directo (de objetos o fenómenos) o declarativo (la expresión, representación, de lo que sabemos). Otra visión del conocimiento, establece una diferenciación bipolar entre el conocimiento tácito y el explícito, tal como ya hemos comentado anteriormente; diferencia que desde nuestro punto de vista, se funda en su nivel de consenso y transferencia, y no tanto en la naturaleza de la fuente.

Según (Gadomski, 1999), los datos son todo aquello que puede ser procesado o transformado mediante procesos computacionales o mentales; mientras que la información son aquellos datos que representan propiedades específicas en el dominio de acción de las personas o agentes artificiales, por tanto, toda información está presente en una fuente. Por su lado, el conocimiento es cualquier propiedad abstracta de una persona o agente artificial que es capaz de procesar o transformar, tanto cuantitativa como cualitativamente, en otro tipo de información o conocimiento (p. ej: procedimiento, instrucción, método, modelo, teoría...), y todo conocimiento tiene presente una referencia a un dominio de aplicación.

Para (Pollock, 2002) los datos son una representación formalizada de hechos, conceptos o instrucciones adecuada para su comunicación, interpretación o procesamiento por personas o medios artificiales; mientras que la información son datos dispuestos en patrones significativos o sintetizados. Por su parte, el conocimiento es información condensada dentro del contexto que es útil para la toma de decisiones y la acción. 
Según (Rowley, 2007), los datos son valores discretos acerca de hechos objetivos u observaciones, que no están estructurados ni procesados y no tienen ningún significado por convención. Sin embargo, según (Choo, 1996), suelen formar parte de sistemas físicos de mayor tamaño (libros, paneles de instrumentos...) que nos pueden dar pistas acerca de qué denotan y cómo deben ser interpretados. La información son datos formateados y estructurados que se pueden definir como una representación de la realidad, siempre que se contextualicen e interpreten respecto a un determinado tema o materia. De alguna manera, la información se manifiesta en signos, y debe tener un significado y relevancia respecto a un propósito para alguien; lo que implica, según (Boddy, et al., 2005) que es la persona receptora del signo la que determina si es información o datos, en base a la posibilidad de una estructuración cognitiva de los datos (Choo, 1996), lo que implica que la diferencia principal entre datos e información no es la estructuración, sino la significación. Finalmente, el conocimiento es información procesada en la mente de las personas que aumenta su capacidad para tomar decisiones y realizar acciones de forma efectiva ${ }^{9}$. Para $($ Choo, 1996) la información se convierte en conocimiento a través de una estructuración de las creencias o de la formación de creencias verdaderas justificadas acerca del mundo.

Para (Davenport and Prusak, 1998) los datos son un conjunto de hechos discretos objetivos sobre acontecimientos; mientras que la información tiene forma en sí misma y significado, está organizada para algún propósito. Los parámetros principales que establecen la transformación de los datos en información son su contexto (propósito) y su categorización (estructura conceptual); adicionalmente, los datos pueden estar procesados (cálculos aplicados sobre ellos) o condensados (resumidos). Para estos autores, el conocimiento, que está orientado a la toma de decisiones y la acción, puede ser tanto fluido como estructurado; es intuitivo y difícilmente traducible en palabras o términos y razonamientos lógicos; y siempre, es poseído (interiorizado) por las personas. Para (Zeleny, 2006) el conocimiento es la coordinación deliberada de la acción, es decir, la capacidad de actuar; lo que significa conocer cómo hacer las cosas de forma correcta y efectiva. Mientras que la información es conocer el qué, la estructura que configuran los conceptos y las relaciones entre las cosas; y los datos, simplemente es no conocer.

Tanto (Schreiber, et al., 1999) como (Uriarte, 2008) coinciden en definir los datos como señales sin significado ni contexto; mientras que para ellos la información son datos que adquieren significado mediante un contexto. El conocimiento es para (Schreiber, et al., 1999) un cuerpo de datos e información que las personas utilizan para tomar decisiones y actuar, presenta por tanto dos grandes diferencias respecto a

${ }^{9}$ Sin embargo, (Ackoff, 1989) establece que el foco del conocimiento es la eficiencia, mientras que el de la sabiduría es la eficacia. 
sus generadores: un propósito y una capacidad generativa, ya que una de las principales facultades del conocimiento es generar nueva información.

Otro rasgo que diferencia al conocimiento de la información es su estabilidad (Uriarte, 2008), es decir, mientras que la información establece patrones relacionales (dependencias) entre los datos, los valores de estos se transforman continuamente, haciendo que la información sea inestable; sin embargo, cuando se presentan correlaciones invariantes en dichos patrones relacionales, y por tanto, somos capaces de entender y predecir su comportamiento la información se transforma en conocimiento, lo que implica que las pautas del modelo del mundo que estamos asumiendo presenten cierta estabilidad respecto al tiempo.

Para (Aamodt and Nygård, 1995), desde el enfoque del proceso de toma de decisiones en el ámbito de la Inteligencia Artificial, los datos son patrones sin significado que son la entrada para un proceso de interpretación que transforma la estructura sintáctica de los datos en una estructura semántica de información en un contexto del mundo real y para un propósito concreto; la información es datos con significado, es la salida del proceso de interpretación y la entrada al proceso de aprendizaje; el conocimiento es información aprendida que se incorpora como recurso activo de razonamiento interiorizado dentro del proceso de decisión de un agente natural o artificial. En este enfoque, el conocimiento cumple tres roles ${ }^{10}$ diferentes dentro del proceso de toma de decisión: $1^{\circ}$ ) se utiliza conocimiento anterior (observaciones anteriores, reglas heurísticas, modelos...) para transformar los datos en información (interpretación), $2^{\circ}$ ) se utiliza conocimiento aprendido (hipótesis demostradas y sus conclusiones, caracterización de casos, excepciones de casos aprendidas, criterios de decisión aprendidos...) para generar nueva información (elaboración), que a su vez, modifica el conocimiento que se utiliza en el proceso de interpretación y $3^{\circ}$ ) se utiliza para adquirir nuevo conocimiento (aprendizaje), es decir, integrar nueva información en un cuerpo de conocimiento, de manera que se hace potencialmente útil de cara a subsiguientes tomas de decisiones; teniendo en cuenta que dicha nueva información puede provenir del aprendizaje de la información recibida como de la elaboración de nueva información. Dos características importantes que diferencian los datos de la información y del conocimiento son el grado de flexibilidad y de integración; mientras que los datos no son flexibles ni están integrados, la información los flexibiliza categorizándolos y los integra relacionándolos, y el conocimiento flexibiliza la información generalizando y reutilizando hipótesis, casos, criterios... al mismo tiempo que se configura como una totalidad integrada.

${ }^{10}$ (Tuomi, 1999) defiende que los datos, en base a la jerarquía del conocimiento invertida, más que ser la base para la creación de la información, y ésta para la generación del conocimiento, emergen una vez el conocimiento y la información han sido relacionados con un problema práctico y específico. 
(Chiang, et al., 1992), desde el ámbito de los Sistemas Basados en Conocimiento, precisan que los datos, la información y el conocimiento requieren los signos para su representación y procesamiento mediante los ordenadores; y realizan un análisis del rol de cada uno de estos elementos en base a los niveles sintáctico, semántico y pragmático del modelo semiótico de Morris (Morris, 1962). En el nivel sintáctico hallamos un conjunto de reglas mediante el cual los signos de un lenguaje se pueden combinar y representar, unos signos son datos, es decir, enunciados atómicos mediante fórmulas bien formadas que denotan hechos individuales, mientras que otros son conocimiento, enunciados de fórmulas bien formadas cuantificadas que denotan hechos acerca de clases; en el nivel semántico hallamos un conjunto de reglas mediante el cual se definen la interpretación y el significado de los signos, de la misma manera, unos signos son datos, que afirman y reflejan las propiedades de una instancia particular (interpretación extensional), mientras que otros son conocimiento, afirmando y reflejando las propiedades de una clase o las relaciones entre las propiedades de diferentes clases (interpretación intensional); en el nivel pragmático hallamos un conjunto de reglas mediante el cual se enlazan los signos con el intérprete (humano o artificial), enlaces que pueden ser puramente funcionales (utilidad del conocimiento respecto a un objetivo ${ }^{11}$ ), o epistémicos (reflexión acerca del conocimiento que se posee y genera, incluyendo la veracidad de los hechos y de las argumentaciones.

\section{Acepciones comunes de los elementos de la Cadena DIC}

En la Tabla 1 resumimos las diferentes definiciones de datos, información y conocimiento en la Cadena DIC. Partimos de las definiciones más amplias o generales, para pasar de un enfoque de la Gestión de Información y la Gestión del Conocimiento, hasta el de la Ingeniería del Conocimiento. En base a las definiciones propuestas por los diferentes autores podemos identificar una serie de acepciones comunes.

${ }^{11}$ Según el principio de racionalidad de (Newell, 1982). 


\begin{tabular}{|c|c|c|}
\hline Fuente & Término & Definición \\
\hline \multirow{3}{*}{$\begin{array}{l}\text { Dicciona- } \\
\text { rio R.A.E. }\end{array}$} & Datos & Antecedente para obtener una conclusión legítima \\
\hline & Información & $\begin{array}{l}\text { Adquisición/comunicación de conocimiento que permite ampliar o precisar el } \\
\text { que ya se posee }\end{array}$ \\
\hline & Conocimiento & Entender la naturaleza, cualidades y relaciones de las cosas \\
\hline \multirow{3}{*}{$\begin{array}{l}\text { Diction- } \\
\text { ary of } \\
\text { Library } \\
\text { and } \\
\text { Infor- } \\
\text { mation } \\
\text { Science }\end{array}$} & Datos & $\begin{array}{l}\text { Hechos representados de forma que pueden ser comprendidos, interpretados y } \\
\text { comunicados }\end{array}$ \\
\hline & Información & $\begin{array}{l}\text { Datos representados de forma comprensible a los que se les ha asignado un } \\
\text { significado en un contexto }\end{array}$ \\
\hline & Conocimiento & $\begin{array}{l}\text { Información que ha sido interiorizada, previamente comprendida y evaluada } \\
\text { en base a la experiencia }\end{array}$ \\
\hline \multirow[t]{3}{*}{ Zins } & Datos & $\begin{array}{l}\text { Evidencias de hechos básicos discernibles y perceptibles codificadas median- } \\
\text { te símbolos }\end{array}$ \\
\hline & Información & $\begin{array}{l}\text { Reificación de datos, convirtiéndolos en signos (interpretados). Organización } \\
\text { de datos basada en una ontología que establece las relaciones relevantes e } \\
\text { invariantes en algún contexto }\end{array}$ \\
\hline & Conocimiento & $\begin{array}{l}\text { Entendimiento interiorizado de la información orientada al uso (acción), que a } \\
\text { veces puede ser representado (exteriorizado). Patrones invariantes de infor- } \\
\text { mación que generan juicios (conclusiones) acerca del mundo. Puede ser } \\
\text { subjetivo (individual, interno, tácito, difícilmente formalizable) u objetivo } \\
\text { (colectivo, externo, explícito, formalizable). Puede ser, desde un punto de } \\
\text { vista epistemológico, práctico (habilidades), directo (fenómenos) o declarati- } \\
\text { vo (representación de lo que se sabe, reflexivo) }\end{array}$ \\
\hline \multirow[t]{3}{*}{$\begin{array}{l}\begin{array}{l}\text { Ga- } \\
\text { domsky }\end{array} \\
\end{array}$} & Datos & $\begin{array}{l}\text { Todo aquello que puede ser procesado o transformado mediante procesos } \\
\text { computacionales o mentales }\end{array}$ \\
\hline & Información & Datos que representan propiedades específicas en el dominio de acción. \\
\hline & Conocimiento & $\begin{array}{l}\text { Propiedad abstracta de un agente capaz de procesar y transformar la informa- } \\
\text { ción en otra información o conocimiento. }\end{array}$ \\
\hline \multirow[t]{3}{*}{ Pollock } & Datos & Representación formalizada de hechos, conceptos o instrucciones \\
\hline & Información & Datos dispuestos en patrones significativos o sintetizados \\
\hline & Conocimiento & $\begin{array}{l}\text { Información condensada en el contexto útil para la toma de decisiones y la } \\
\text { acción }\end{array}$ \\
\hline \multirow[t]{3}{*}{ Rowley } & Datos & Valores discretos de hechos objetivos básicos sin significado por convención \\
\hline & Información & $\begin{array}{l}\text { Datos formateados y estructurados, contextualizados e interpretados (con } \\
\text { significado) que son representación de la realidad. }\end{array}$ \\
\hline & Conocimiento & Información procesada que permite tomar decisiones y realizar acciones \\
\hline \multirow[t]{3}{*}{ Choo } & Datos & Hechos o mensajes \\
\hline & Información & Datos con significado en base a su estructuración cognitiva \\
\hline & Conocimiento & $\begin{array}{l}\text { Estructuración y formación de creencias verdaderas justificadas en base a la } \\
\text { información }\end{array}$ \\
\hline \multirow{2}{*}{$\begin{array}{l}\text { Davenport } \\
\text { y Prusak }\end{array}$} & Datos & Hechos discretos objetivos \\
\hline & Información & $\begin{array}{l}\text { Datos con forma (categorización) y significado (dependencia, correlación) } \\
\text { respecto algún propósito }\end{array}$ \\
\hline
\end{tabular}




\begin{tabular}{|c|c|c|}
\hline & Conocimiento & Información útil para la toma de decisiones y la acción \\
\hline \multirow[t]{3}{*}{ Uriarte } & Datos & Símbolos sin contexto \\
\hline & Información & $\begin{array}{l}\text { Entendimiento de las relaciones existentes entre los datos dependientes en un } \\
\text { contexto }\end{array}$ \\
\hline & Conocimiento & $\begin{array}{l}\text { Entendimiento de los patrones de relación entre datos e información y de sus } \\
\text { implicaciones en un nuevo contexto }\end{array}$ \\
\hline \multirow[t]{3}{*}{$\begin{array}{l}\text { Aamodt y } \\
\text { Nygard }\end{array}$} & Datos & $\begin{array}{l}\text { Patrones sintácticos sin significado pragmático (para un contexto y propósito } \\
\text { concretos) }\end{array}$ \\
\hline & Información & Datos interpretados con significado (mediante conocimiento) \\
\hline & Conocimiento & $\begin{array}{l}\text { Información aprendida, o elaborada, e interiorizada que se convierte en } \\
\text { recurso activo de conocimiento para la toma de decisiones }\end{array}$ \\
\hline \multirow{2}{*}{$\begin{array}{l}\text { Chiang, } \\
\text { Barron y } \\
\text { Storey }\end{array}$} & Datos & $\begin{array}{l}\text { Enunciados atómicos que denotan hechos individuales con interpretación } \\
\text { extensional }\end{array}$ \\
\hline & Conocimiento & $\begin{array}{l}\text { Enunciados cuantificados que denotan hechos de clases con interpretación } \\
\text { intensional, argumentos y utilidad }\end{array}$ \\
\hline \multirow{3}{*}{$\begin{array}{l}\text { Schreiber } \\
\text { y Cols. }\end{array}$} & Datos & Señales no interpretadas \\
\hline & Información & Datos provistos de significado \\
\hline & Conocimiento & $\begin{array}{l}\text { Conjunto de datos e información que las personas utilizan para apoyar la toma } \\
\text { de decisiones y la acción }\end{array}$ \\
\hline
\end{tabular}

Tabla 1. Definiciones de datos, información y conocimiento desde las perspectivas de la Gestión de la Información, la Gestión del Conocimiento y la Ingeniería del Conocimiento.

Aunque cada autor aporta su matiz a la definición de dato, yendo desde un simple estímulo sensorial hasta una representación formalizada, todos giran en torno a la concepción de que los datos no son más que la antesala, la materia prima, para obtener la estructuración-significación de los mismos en información. Agregando las acepciones más comunes, se puede decir que los datos son evidencias de hechos discernibles, discretos (que no implica que sean atómicos sino instancias) y objetivos, obtenidos de la observación (de un dispositivo o mediante la percepción directa) y que sirven de antecedente para obtener una conclusión legítima.

En cuanto al concepto de información las diversas acepciones más comunes aportadas por los diferentes autores antes referenciados las podemos agregar como la reificación de datos que se convierten en signos con forma (representación y estructura) y con significado (interpretación) en base a su conexión relacional (dependencias) y su estructura cognitiva (categorías), en un contexto y para alguien (o algo), sobre la que se pueden identificar patrones de concordancias presentes en los datos (las instancias).

En general las definiciones aportadas sobre el concepto de conocimiento hacen hincapié en la toma de decisiones como su fin último para afirmar que estamos ante conocimiento y no información. No obstante, y para completar la definición, podemos agregar las múltiples acepciones (no tan comunes) de conocimiento como el entendimiento (interiorización por alguien o algo) mediante la estructuración y formación de creencias verdaderas justificadas acerca de la naturaleza, cualidades y relaciones de 
las cosas (modelo), en base a la identificación de patrones invariantes de concordancias presentes en los datos embebidos en la información (experiencia en un contexto), obteniendo generalizaciones (y excepciones) que soportan la toma de decisiones y la acción.

\section{Análisis de los elementos de la Cadena DIC desde el pragmatismo}

Para (Boddy, et al., 2005), es la persona, como intérprete de los hechos que le rodean, la que determina si son información o datos en base a la posibilidad de su estructuración cognitiva; lo que depende del significado y relevancia que dichos hechos representan en relación a un propósito (Choo, 1996); decisiones que se apoyan en el principio de racionalidad limitada (Simon, 1972). Por otro lado, (Low, 2009) critica tomar como punto de partida el modelo lineal de la Cadena DIC (Zins, 2007), proponiendo que los datos forman parte de la categoría peirceana de primeridad, la información de la de segundidad y el conocimiento de la de terceridad ${ }^{12}$.

En estas precisiones y crítica podemos observar que se mencionan dos cuestiones importantes: la significación como proceso que realiza el intérprete de los hechos (fenómenos) y la categorización de la naturaleza de los fenómenos. Por ello debemos contextualizar y precisar dichos conceptos y seguidamente profundizar en sus cualidades; y de esta manera revisar la Cadena DIC desde otro enfoque, el de la semiótica; enfoque que nos permitirá inicialmente identificar las imprecisiones y deficiencias de las definiciones establecidas en las fuentes analizadas, y finalmente, establecer de forma más precisa las diferencias, los roles y las dependencias de sus componentes.

Para entender el concepto de signo en (Peirce, 1905) debemos contextualizarlo en su filosofía pragmatista ${ }^{13}$, y en concreto dentro de un enfoque empirista, mediante el cual la realidad, el mundo, no puede ser concebida al margen de sus representaciones o fenómenos mentales. Por tanto, el conocimiento pende de su representación, los signos, para la obtención de cualquier deducción, y también inducción o abducción, apoyado en la experiencia; signos que son de naturaleza fenomenológica, es decir, la representación de los conceptos universales presentes en los fenómenos. Los conceptos son clases que hacen emerger el ser: la unidad en la cualidad, la dualidad en la relación, y la trinidad en la representación ${ }^{14}$; de la substancia: lo variado y múltiple de

\footnotetext{
${ }^{12}$ Lo que (Castañares, 1985) traduce como cualidad, realidad y ley, en los fenómenos aplicados a los objetos.

${ }^{13}$ Que él denominó finalmente pragmaticismo, "pues es lo suficientemente fea como para quedar a salvo de secuestradores” (Peirce, 1905).

${ }^{14}$ Porque según (Castañares, 1985) todo juicio, que es el acto por el que conocemos, es la aplicación de un predicado (cualidad) a un sujeto (instancia); dicha cualidad la conocemos por contraste o semejanza entre un relato y un correlato (instancias discernibles), relación binaria
} 
los sujetos, las instancias particulares. Además, y esto es lo que enlaza el signo con el pragmatismo, es que el conocimiento, como entendimiento de la realidad ${ }^{15}$, consiste en la construcción de creencias, y toda creencia entraña un hábito, que se constituye como criterio de diferenciación de ésta (Peirce, 1867). Lo que el hábito define es el cuándo y el cómo de nuestra acción; el cuándo es cualquier estímulo para la acción y el cómo su propósito o resultado.

(Peirce, 1897) define un signo o representamen, como algo que, para alguien (intérprete), representa o se refiere a algo (objeto ${ }^{16}$ ) en algún aspecto o carácter (fundamento), creando en ese alguien un signo equivalente (interpretante) más desarrollado. Por tanto, un signo es algo que sirve para transmitir el conocimiento de otra cosa, lo que implica que lo ha de representar. En esta definición del signo debemos tener especial cuidado con el interpretante; ya que este puede ser inmediato, el efecto total no analizable producido por el signo; dinámico, el efecto que un emisor se propone producir mediante el signo y el producido en un receptor; o final, el efecto que el signo produciría sobre cualquier receptor (intérprete) en aquellas circunstancias en las que el signo ejerciera su pleno efecto.

Según (Castañares, 1994), para Peirce un signo es de un tipo dependiendo de: a) su propia posibilidad de representación (representamen), en relación al objeto que representa (objeto), o en relación a lo que interpreta de él (interpretante) su receptor y; b) en relación a los fenómenos ${ }^{17}$ que representa, es decir, una cualidad, un hecho o una ley.

que debe presentarse invariante para poder establecer la cualidad; y dicha comparación solo es posible mediante una representación.

${ }^{15}$ Realidad que es entendida por el pragmatismo como los peculiares efectos sensibles que producen las cosas que participan en ella.

${ }^{16}$ Que puede ser una cosa singular existente, o que se cree que ha existido o que se espera que exista, o una cualidad o relación o hechos conocidos, o algo de naturaleza general invariablemente encontrado en ciertas circunstancias.

${ }^{17}$ Para Pierce el primer elemento común a todo fenómeno es su puro presentarse sin referencia a cualquier otra cosa; algo que no puede ser analizado ni comparado, una cualidad o un sentimiento, como la idea abstraída de un color sin tener en cuenta su relación con un objeto concreto (p. ej.: la blancura, lo oxidado). El segundo elemento de todo fenómeno es su imposición insoslayable sobre nuestra experiencia; es la categoría de lo existente de lo factual, nos hace conscientes de lo otro, implica acción y reacción, causa y efecto; por tanto se relaciona con la causalidad del mundo físico (p. ej.: un agujero en una puerta nos indica que algo la ha golpeado). Por último, en cuanto descubrimos la ley o razón por la cual sucede cualquier fenómeno aparece su tercer elemento; es la categoría de la razonabilidad y la representación, implica regularidad y hábito, hace inteligible la experiencia bruta de lo factual (p. ej.: manejar una ventana bruscamente implica que su durabilidad se reduzca sensiblemente). A estos tres elementos los denomina, primeridad, segundidad y terceridad, respectivamente (Redondo, 2009, p. 175-178). 
De esta manera (Peirce, 1903), como representamen, un signo puede ser un cualisigno, un sinsigno o un legisigno ${ }^{18}$. El cualisigno es la representación de la pura cualidad o idea sin actualidad, es decir, no relacionada con ningún objeto e independiente de que sea percibida o recordada (p. ej.: el color rojo o la rojez, no la rojez concreta de ese papel que tengo sobre la mesa), es una representación abstracta, es la forma de la forma. El sinsigno (que viene de signo singular, simple) es la representación de lo fáctico actual, es decir de los hechos concretos e individuales impuestos a nuestra experiencia y que nos hacen ser conscientes del mundo (lo otro) (ahora sí, p. ej.: la rojez concreta de ese papel rojo de la libreta que está sobre mi mesa), es la forma de la existencia. Por último, el legisigno es la representación de una ley ${ }^{19}$ por la cual se establece el significado de sinsignos, por tanto convencional y general, que no es representación de un objeto único sino de un tipo significante (p. ej.: podría establecer en mi puesto de trabajo que todos los papeles rojos indican datos peligrosos, $\mathrm{y}$ bajo esta ley, todos los papeles rojos de mi despacho se convierten en sus instancias), es la forma de la necesidad.

Por otro lado, en su relación con el objeto, un signo puede ser un icono, un índice o un símbolo. Un icono es un signo que mantiene una relación de semejanza o similitud con el objeto (p.ej.: una fotografía o un plano (de cualidades), un diagrama (de relaciones), una metáfora ${ }^{20}$ (paralelismo)). Un índice se refiere a un objeto por el que está afectado, el signo está en conexión dinámica con el objeto, carece de todo parecido con el objeto, es factual, es existencial y dirige la atención al objeto que es un hecho (p. ej.: la temperatura corporal elevada de un enfermo de gripe, o la veleta de viento al girar a cierta velocidad); por tanto, debe poseer cualisignos que hacen de representación de la conexión dinámica con el objeto, lo que involucra que también posea iconos, iconos que no están relacionados por semejanza con el objeto, sino con el cambio en el objeto que obra sobre el índice (p. ej.: la diferencia de graduación de la rojez de la piel nos podría indicar el nivel de picadura de un mosquito). En tercer lugar, un símbolo es un signo que determina a un interpretante en virtud de una ley o convención, toma del objeto algún aspecto general que se convierte en la interpretación del signo, lo que sirve para explicitar el significado general de todas sus réplicas (instancias) (p. ej.: la palabra "persona" hace referencia a la clase persona con sus atributos comunes a todas sus instancias, los individuos; o el símbolo de la cruz roja es un icono que complementa al logotipo "Cruz Roja”, transformando, por conven-

${ }^{18}$ (Eco, 1988, p. 55) denomina al cualisigno, sinsigno y legisigno, tone, token y type respectivamente; es decir, la cualidad de un signo, una cosa o acontecimiento que existe fácticamente y que es un signo, y el modelo abstracto de un sinsigno.

${ }^{19}$ Un legisigno, por lo general, requiere de réplicas (instancias) para formarse y actualizarse, que son sinsignos, signos factuales, que soportan una inducción.

${ }^{20}$ Lo que muestra la flexibilidad del signo icónico. 
ción, el significado de la cruz, en el de ayuda o socorro); significando solo en base a éstas.

Finalmente, en su relación con el interpretante, un signo puede ser un $r h e m a^{21}$, un dicisigno o un argumento. Un rhema es un signo que genera en el intérprete un interpretante íconico o índice degenerado abstractos (p. ej.: la cualidad "es rojo", que es el término de una proposición, puede causar en el intérprete una imagen de rojo o crear una reacción sentimental respecto al rojo, y en este último caso, posiblemente el interprete relacione el rojo con situaciones dolorosas o peligrosas), es decir, dirige al el interpretante del objeto hacia una cualidad determinada. Un dicisigno es un signo que indica el sentido de un rhema con su referente ${ }^{22}$, determinando al interpretante respecto a la información que posee el signo; pudiendo ser de tipo modal, los que articulan lo posible, o existencial (p. ej.: la frase "Este es un texto acerca de la Cadena DIC”, o "Ese niño del gorro"). Por último, un argumento es un signo cuyo interpretante es una ley, es decir, la conexión sistemática, legal e inferencialmente a otros signos (dicisignos), que puede ser abductiva, inductiva o deductiva (p. ej.: "Si los papeles rojos de mi despacho se refieren a datos peligrosos y encuentro un papel rojo, deduzco que los datos que contiene son peligrosos" es un argumento deductivo).

Sin embargo, estas tres tricotomías no se refieren a un signo en su conjunto, sino a algún aspecto de éste; por tanto, para referirnos a la significación como proceso de significar respecto a un signo, debemos de tener en cuenta los tres aspectos del signo (representamen, objeto e interpretante). De esta manera, Peirce define diez clases de signos en tres categorías (Magariños, 2008, p. 146-150):

\section{Semiótica icónica}

- $\quad \mathbf{1}^{\text {a }}$ Cualisigno (icónico rhemático): toda cualidad (tone) en la medida en que es un signo (p. ej.: la sensación inmediata del rojo, que muchas veces se utiliza como icono (réplica) de la connotación del rhema peligro; o la sensación inmediata de la temperatura). Un cualisigno nos enlaza como intérpretes a los aspectos perceptuales del objeto representados en el signo.

- $\quad 2^{\mathrm{a}}$ Sinsigno icónico (rhemático): todo icono concreto y factual (token) como esencia/idea de un objeto (p. ej.: un triángulo concreto que se utiliza como réplica del concepto triángulo; una caricatura de Ernest Hemingway; o una fotografía de la Catedral de Notre Dame). Un sinsigno icónico nos enlaza como intérpretes a los aspectos formales del objeto representados en el signo.

- $\quad 3^{\mathrm{a}}$ Legisigno icónico (rhemático): el icono (genérico) como esencia conceptual (type) (p. ej.: el diagrama topológico de cualquier triángulo, o el teorema

\footnotetext{
${ }^{21}$ Este tipo de signo es denominado sema en la semántica lingüística.

${ }^{22}$ Lo que denominaríamos proposición.
} 
de Pitágoras). Un legisigno icónico nos enlaza como intérpretes a los aspectos formales generales del objeto representados en por el signo.

\section{Semiótica indicial}

- $\quad 4^{a}$ Sinsigno indicial rhemático: el índice factual (token) que dirige la atención al objeto que causa su presencia (p. ej.: el grito espontáneo /¡coche!/; o el sonido de un instrumento musical).

- $\quad 5^{\text {a }}$ Sinsigno (indicial) dicisigno: el índice factual (token) como suministrador de información en un contexto dado (p. ej.: la veleta de un campanario nos suministra información acerca de la dirección y la velocidad del viento en un momento dado; o el termómetro que marca la temperatura de un paciente). Un sinsigno dicisigno nos enlaza como intérpretes al estado del objeto en un contexto representado por el signo.

- $\mathbf{6}^{\mathbf{a}}$ Legisigno indicial rhemático: el índice como esencia de un comportamiento general (type) (p. ej.: el pronombre demostrativo /este/; o los colores de un semáforo). Un legisigno indicial rhemático nos enlaza como intérpretes a la norma representada perceptualmente por el signo.

- $\quad 7^{a}$ Legisigno indicial dicisigno: el índice como suministrador de información de un comportamiento general (type) (p. ej.: el funcionamiento del indicador de cualquier termómetro de mercurio). Un legisigno inidicial dicisigno nos enlaza como intérpretes a la norma representada contextualmente por el signo.

\section{Semiótica simbólica}

- $\quad 8^{a}$ Símbolo rhemático (legisigno): el concepto general (type) que, por convención, determina las cualidades comunes de diversos sinsignos indiciales rhemáticos (p. ej.: el concepto mesa agrupa y resume las cualidades, y los comportamientos asociados de uso, de todas las mesas existentes; cualquier nombre común; o la bandera de un país). Un símbolo rhemático nos enlaza como intérpretes a algún aspecto de generalidad del objeto, por hábito, representado por el signo.

- $9^{a}$ Símbolo (legisigno) dicisigno: la declaración de relaciones (legisignos icónicos rhemáticos) entre conceptos (símbolos rematicos legisignos) (types) que nos suministra información (p. ej.: la proposición declarativa "La libreta de mi mesa tiene papeles rojos”). Un símbolo dicisigno nos designa como intérpretes a una asociación de ideas y relaciones generales conectadas al objeto.

- 10 ${ }^{\mathrm{a}}$ Argumento (legisigno simbólico): la declaración de la ley (type) que prescribe la coexistencia de un conjunto de símbolos legisignos dicisignos (p. ej.: cualquier argumento abductivo, inductivo o deductivo). Un argumento 
nos enlaza como intérpretes a los patrones invariantes de concordancia que se presentan en un conjunto de proposiciones.

Por otro lado, debemos precisar y aclarar el proceso de significación, denominado semiosis, palabra que toma Peirce de Filodemo de Gadara, epicúreo del siglo I a.C., para definir la "inferencia sígnica". Como reitera en muchos textos suyos, para Peirce, la lógica es la ciencia de las condiciones necesarias de consecución de la verdad, que en sentido amplio, se ocupa de las leyes del pensamiento (conocimiento); pero como éste solo es posible a través de los signos, la lógica ha de ser una semiótica (Peirce, 1902). Por tanto, la lógica, a nivel semiótico, es el análisis de los argumentos legisignos simbólicos, apoyados en los símbolos rhemáticos legisignos y los símbolos legisignos dicisignos. Dicho análisis se apoya en el proceso de la semiosis (la constitución de significado) ilimitada a través de los interpretantes inmediato, sucesivos dinámicos y final; es decir, tal como especificábamos en la agregación de las definiciones más comunes del conocimiento, los patrones invariantes de concordancias presentes en los datos embebidos en la información, obteniendo generalizaciones que soportan nuestras decisiones y acciones, de forma legítima.

Entonces, (Low, 2009) nos propone que los datos forman parte de la categoría de primeridad, la información de segundidad y el conocimiento de terceridad. Sin embargo, si tenemos en cuenta las definiciones más comunes (en base a la agregación propuesta) de los conceptos datos, información y conocimiento, podemos observar: $1^{\circ}$ ) que el cualisigno icónico rhemático es el único signo completo de primeridad, siendo solo una cualidad de un hecho discernible, discreto y objetivo obtenido de la observación que sirve como antecedente de una conclusión legítima; $2^{\circ}$ ) los sinsignos, en sus diferentes variantes, todos ellos signos de segundidad, no son la reificación de los datos convertidos en algo con forma y significado en un contexto y para un intérprete o receptor; $3^{\circ}$ ) solo los legisignos y los símbolos, en sus diferentes vertientes, todos ellos signos de terceridad, nos muestran el conocimiento, como el entendimiento conceptual, relacional y causal de un modelo acerca del mundo, entendimiento que es de las invariantes presentes en los tres componentes del modelo; y $4^{\circ}$ ) el autor no identifica, de forma explícita, el rol de los signos de terceridad como mecanismos (el conocimiento necesario) de estructuración de la información y de reificación de los datos; identificación que si realizan (Aamodt and Nygård, 1995).

Por su parte, (Nake, 2002) defiende que los datos son signos reducidos a la dimensión sintáctica, el aspecto material de la información; mientras que la información está compuesta por signos reducidos a la dimensión semántica, la relación convencional entre el representamen y el objeto; y por último, el conocimiento lo está por signos plenos en su relación triádica representamen-objeto-interpretante en la dimen- 
sión pragmática. (Beynon-Davies, 2009) establece, en base a (Stamper, et al., 2000) ${ }^{23}$, cuatro ${ }^{24}$ niveles de sistemas de signos: $1^{\circ}$ ) el nivel pragmático, en el que las intenciones enlazan los signos con la acción; $2^{\circ}$ ) el nivel semántico, en el que se enlazan los signos con su significado; $3^{\circ}$ ) el nivel sintáctico, en el que se enlazan los signos con el formalismo utilizado para su representación (contexto gramatical); y $4^{\circ}$ ) el nivel empírico, en el que se establece la codificación de los signos (los datos).

Vamos a utilizar un ejemplo de (Schreiber, et al., 1999), que nos va a servir para esclarecer ciertas confusiones muy comunes, en relación a las conceptualizaciones acerca de los datos, la información y el conocimiento, desde el enfoque pragmaticista de Peirce acerca de la semiosis y los signos.

En este ejemplo, basado en el código Morse, los autores de CommonKADS ${ }^{25}$ establecen la definición y relación entre datos, información y conocimiento (la Cadena DIC) de la siguiente manera: los datos en bruto, no interpretados, son la cadena de símbolos “...---...”; S.O.S. es el significado asignado a los datos, la información; y "Alerta de emergencia implica comenzar operación de rescate" es la fijación de relevancia y propósito sobre la información, destinados a tomar una decisión o ejecutar una acción, el conocimiento.

En el ejemplo se ratifica la concepción de los datos como meros símbolos (no en el sentido semiótico) con estructura sintáctica sin significado, y de la información como asignación de significado a los datos respecto a un contexto específico y para alguien. Sin embargo, tal como hemos visto en la semiótica de Peirce, “...---...” es una cadena de iconos que conforman la declaración (dicisigno) de un índice (hecho factual), que se convierte en símbolo para el receptor del mensaje, argumento de la traducción a la cadena "S.O.S", cadena que ejerce como interpretante inmediato en la cadena semiósica, generando el interpretante dinámico "Alerta de emergencia implica comenzar operación de rescate", que a su vez, continuando con la cadena inferencial de la semiosis, genera el interpretante final de la acción "Ir al rescate". Por tanto, el ejemplo realiza toda una serie de saltos semiósicos ignorando ciertos signos, y sus tipos, que intervienen en la supuesta "simple" Cadena DIC: $1^{\circ}$ ) el receptor ha de disponer de los rhemas (terceridad, conocimiento) que establecen la combinatoria del código morse y de los argumentos (terceridad, conocimiento) que traducen el código morse al lenguaje natural; $2^{\circ}$ ) resuelta la traducción, la cadena "S.O.S", sigue presentando la complejidad de requerir del argumento convencional que convierte el índice, que a su vez es un dicisigno, en símbolo (de peligro); y $3^{\circ}$ ) una vez establecido el signo como

\footnotetext{
${ }^{23}$ Realmente, en (Stamper, 1996) se definen cinco niveles de sistemas de signos, identificando los signos (o tokens) físicos, como entrada del nivel empírico.

${ }^{24}$ Siendo los tres primeros, como ya hemos visto, los definidos por (Morris, 1962).

${ }^{25}$ Es el estándar europeo utilizado en ingeniería del conocimiento para el modelado de sistemas basados en el conocimiento (BSC), <<http://commonkads.org >>.
} 
símbolo, interpretante inmediato de la cadena semiósica, se requiere del argumento general que enlaza el dicisigno con el comportamiento esperado, generado sobre las evidencias de las instancias concretas y diversas (i. e. : "Cualquier llamada de peligro a) indica un peligro y b) requiere de una acción que lo anule o mitigue”).

Ahora vamos a analizar un segundo ejemplo, de los mismos autores, que explicita la tarea de diagnóstico médico diferencial de CommonKADS ${ }^{26}$. En el diagrama de inferencias de la tarea, un dato, $37^{\circ} \mathrm{C}$, se relaciona con la temperatura de un paciente en el contexto del diagnóstico médico; de esta manera, se describe (el valor de) una variable de estado o síntoma del paciente, convirtiéndose así en información que posteriormente, mediante una inferencia denominada Abstraer, se transforma en la categoría "Fiebre", etiqueta de un síntoma anormal; transformación que se realiza mediante un conocimiento de clasificación. Una vez se dispone de dicha etiqueta, se identifican las diferentes explicaciones o causas (por abducción) que la pueden generar mediante la inferencia Cubrir, para seguidamente, hallar las evidencias de los síntomas requeridos de cada explicación en el paciente, y de esta manera, mediante diferenciación, seleccionar como candidata aquella explicación en la que están presentes todos los síntomas (la mejor explicación), descartando las demás. En este ejemplo ya no se sigue con la concepción de los datos como meros componentes sintácticos, pero sí de la información como asignación de significado contextual a éstos.

Vamos a analizar la entrada y salida de la inferencia Abstraer desde el enfoque de la semiosis y los signos. La temperatura concreta del paciente, como hecho observable directamente, es un sinsigno rhemático indicial que nos aporta el estado de un aspecto relevante del paciente, compuesto por el cualisigno que es la sensación de dicha cualidad, el sudor provocado por la alta temperatura; pero como dicha temperatura solo la podemos evidenciar de manera precisa mediante un instrumento, un termómetro, se convierte en un sinsigno dicisigno; por otro lado, si esa temperatura estuviera registrada en algún soporte, sería un símbolo dicisigno. En el primer caso, no tendríamos un signo (evidencia) preciso, es decir, observaríamos el sudor del paciente (cualisgino), funcionando como representación de cambio y componente de un índice, un signo de segundidad; en el segundo caso, tendríamos un signo preciso, el indicador de temperatura del termómetro, funcionando como índice que informa acerca del síntoma del paciente mediante un cualisigno, un signo de terceridad; en el tercer caso el signo es la representación externa (interpretante) del hecho de forma declarativa, en la que identificamos explícitamente los conceptos "paciente" y "temperatura" cuyas instancias serían el paciente concreto y el valor de $37^{\circ} \mathrm{C}$, conectados mediante la relación “Tiene Temperatura” (i. e. : “Tiene_Temperatura(Paciente Y, 37

\footnotetext{
${ }^{26}$ Que se basa en la tarea genérica de diagnóstico mediante abducción de (Chandrasekaran, et $a l .$, 1992).
} 
$\left.{ }^{\circ} \mathrm{C}\right)$ ") transcrito, por ejemplo, en lenguaje natural, un signo de terceridad. En estos tres casos se realiza una sola semiosis, o proceso de significación, la primera en esta cadena inferencial semiósica. Por otro lado, el signo que nos muestra la categoría "Fiebre" es el resultado de una segunda semiosis, que apoyado en el interpretante inmediato de la primera semiosis (la entrada a la inferencia Abstraer), mediante un argumento inductivo (una categoría), genera un interpretante dinámico (la salida de la inferencia Abstraer).

Una vez analizados los conceptos de signo y semiosis, y su aplicación a los dos ejemplos expuestos, podemos esclarecer las deficiencias identificadas en las propuestas de (Beynon-Davies, 2009; Low, 2009; Stamper, et al., 2000).

(Low, 2009) nos propone que los datos forman parte de la categoría primeridad, la información de segundidad y el conocimiento de terceridad. Sin embargo, si recuperamos la definición agregada de datos, éstos son las evidencias de hechos discernibles, discretos, que se pueden observar, por tanto son signos de segundidad (su conexión con el objeto). También nos dice que la información forma parte de segundidad, cuando a nivel semiótico la información es la declaración, explicitación, de los datos, es decir, son signos de terceridad. Por último, declara que el conocimiento forma parte de la terceridad, y ya que los dicisignos y los símbolos son signos que se relacionan con el interpretante, la tercera propuesta es correcta.

Por su parte, (Nake, 2002) nos propone que los datos son signos reducidos a la dimensión sintáctica, afirmación errónea si tenemos en cuenta que los hechos (signos conectados al objeto) no disponen de dicha dimensión, es la información, entendida como formada por legisignos y símbolos, la que se configura a partir de una sintaxis que, además, declara y representa la semántica de los factuales sinsignos compuestos por cualisignos (hechos y cualidades); y éstos ya disponen de su propia semántica; por tanto, tanto los datos como la información disponen de la dimensión semántica, su diferencia radica en que los primeros son hechos observables y los segundos son declaraciones (y representaciones) de dichos hechos. Por último, nos dice que el conocimiento son signos plenos en la dimensión pragmática, afirmación parcial, ya que lo pragmático tiene relación con el interpretante final del signo (el hábito de decisión y acción del intérprete), mientras que el interpretante inmediato y los dinámicos lo que hacen es construir, dinámica y contextualmente, la cadena inferencial semiósica, compuesta por interpretaciones de interpretaciones que se generan principalmente en la dimensión semántica (significaciones de significaciones).

Por último, (Beynon-Davies, 2009), parte de los tres niveles de los signos (ámbitos) de la semiótica conductista de (Morris, 1962): sintáctico, semántico y pragmático, agregándole el nivel empírico (alimentado a su vez del nivel físico); clasificación que no es cuestionable si de lo que partimos es de signos de la terceridad, es decir, conectados al interpretante; pero si lo que hacemos es partir de los propios hechos (los datos), signos de la segundidad (conectados al objeto), los niveles semántico y empírico forman parte integrante de esos signos, mientras que la sintaxis forma parte de la declaración (representación) de éstos mediante signos de la terceridad (dicisignos y 
símbolos). Finalmente, como en la propuesta de (Nake, 2002), el nivel pragmático solo tiene relación con los intepretantes finales dentro de la cadena inferencial semiósica.

\section{Definición de los elementos de la Cadena DIC desde el pragmatismo}

Basándonos en las acepciones más comunes sobre los elementos de la Cadena DIC, y en el análisis que hemos realizado desde el pragmatismo de Peirce, vamos a proponer una nueva definición. Para ello, partimos de una interpretación de los niveles de sistemas de signos de (Stamper, 1996) y las metáforas de (Zeleny, 2006).

- El mundo físico o de los fenómenos (no saber): en este nivel nos encontramos con los hechos discernibles y discretos (fenómenos), y sus evidencias obtenidas mediante la observación directa o cualquier instrumento de medida; es decir, los datos. La diferencia entre la observación directa y la medida instrumental radica en el nivel de precisión y exactitud de la medición. Por tanto, estamos hablando de sinsignos compuestos por cualisignos (p. ej. el sudor de un paciente con fiebre; el color de un metal incandescente; o la temperatura y humedad en una sauna), y sinsignos dicisignos (p. ej.: la temperatura de un paciente en un termómetro; la presión atmosférica en un barómetro; o el valor EV de un fotómetro TTL enfocando a un paisaje). Al hablar de sinsignos, estamos tratando todos aquellos signos que están causalmente conectados con sus objetos, de manera que nos representan aquellos aspectos relevantes de los fenómenos del mundo. Tal como hemos ido demostrando desde el análisis pragmaticista, el dato, como evidencia de un hecho o fenómeno, es un signo; y como tal, es una representación (representamen) con significado (interpretante) enlazado causalmente (por el argumento del signo) con dicho hecho o fenómeno (objeto); no un mero patrón sintáctico. Sin embargo, cualquier dato lo es de un hecho aislado, es una instancia; su limitación consiste precisamente en que de un único hecho no se puede extraer ninguna conclusión legítima, ya que es inviable identificar ningún patrón invariante de concordancia con otros hechos similares, al no disponer de una estructura que nos permita agruparlos y compararlos.

- El mundo informacional o empírico (saber qué): en este nivel nos encontramos con la reificación de los datos, con forma y estructura, que nos permite identificar patrones de concordancias (categorizaciones y dependencias relacionales) entre estos. Por tanto, estamos hablando de símbolos rhemáticos y símbolos dicisignos (p. ej.: conceptos, atributos, relaciones y declaraciones de todos ellos). Al hablar de estas dos categorías de símbolos, estamos tratando con las explicitaciones de forma y estructura, que nos permiten configurar los cimientos sobre los cuales cosificar los fenómenos y registrarlos. La información, como forma y estructura que permite el registro empírico, y agrupación 
de los datos, se convierte en el soporte explícito y declarativo de la experiencia.

- El mundo del conocimiento (saber cómo): en este nivel nos encontramos con el entendimiento (estructuración y formación de creencias verdaderas y justificadas) de la naturaleza, cualidades y relaciones de un modelo del mundo, mediante la identificación de invariantes de concordancias presentes en los datos (instancias) embebidos en la información (forma y estructura), obteniendo generalizaciones (experiencia) que soportan la toma de decisiones y la acción. Por tanto, estamos hablando de argumentos ${ }^{27}$ (abductivos, deductivos o inductivos) que declaran la ley que prescribe la coexistencia de un conjunto de símbolos rhemáticos y símbolos dicisignos (p. ej.: la primera ley de Newton; o la correlación entre $\mathrm{n}^{\circ}$ de accidentes de coche y el uso del móvil). Hemos de añadir que: a) el conocimiento también participa en la configuración de la forma y estructura del mundo informacional (p. ej.: cuando explicitamos la forma y estructura de un concepto cualquiera para posteriormente cosificar y registrar algún fenómeno estamos haciendo uso del conocimiento, que previamente debe haber construido argumentos extrapolables a la estructura informacional que estamos definiendo); y b) el conocimiento también permite generar nuevos datos en una estructura informacional (p. ej.: cuando utilizamos un argumento inductivo para clasificar un fenómeno estamos generando una nueva instancia de etiqueta de categoría de dicho fenómeno; y cuando utilizamos un argumento deductivo sobre unos datos antecedentes estamos generando nuevos datos de tipo consecuente).

- El mundo de la acción (saber por qué): en este nivel nos encontramos con el conocimiento que genera la acción. Por tanto, estamos hablando de la cadena inferencial basada en la semiosis ilimitada (Bonfantini, 1984). Una semiosis inicial genera un interpretante inmediato que activa una cadena de semiosis encadenadas con interpretante dinámicos, que finalmente activan un interpretante final, la acción del intérprete (p. ej.: cuando voy a hacer gestiones a un edificio de la administración pública, la escala y forma (cualisignos formales, interpretantes inmediatos) de la puerta principal, en relación a otras puertas conocidas por mi experiencia (interpretantes finales simbólicos), o la presencia de un alero, me permiten identificarla como entrada principal; y sus materiales (cualisignos indiciales, interpretantes inmediatos) me ayudan a in-

\footnotetext{
${ }^{27}$ Esta definición aclara que el conocimiento no es solo “información procesada desde un punto de vista mediante representación [...] un hecho codificado que puede ser almacenado, recuperado y transferido" (Nitecki, 1985); por que el conocimiento, aunque pueda ser representado mediante un lenguaje formal, dicho lenguaje es un medio para representar la sustancia del conocimiento: los argumentos.
} 
terpretar (argumentos, interpretantes dinámicos) y deducir (más argumentos, interpretantes dinámicos) qué tipo de fuerza y método de apertura debo aplicar para poder abrirla, y finalmente actuar adecuadamente (interpretante final).

La articulación de la Cadena DIC se genera dinámicamente mediante el proceso de significación de la semiosis ilimitada; en una cadena inferencial que parte de los datos, como instancias de evidencias con significado de los fenómenos; registrados, representados, estructurados y agrupados en el soporte explícito y declarativo de la estructura informacional; sobre la que identificamos patrones invariantes de concordancias que nos permiten generar conocimiento en forma de argumentos sobre categorías, deducciones o hipótesis causales; conocimiento que, a su vez, reconfigura la estructura informacional y genera nuevos datos (modificando el propio conocimiento); conocimiento que es invocado mediante los interpretantes dinámicos en el proceso de significación activado por los interpretantes inmediatos de los datos, en un momento dado, en un contexto concreto, para un intérprete y un propósito específicos; generando una acción resultante como interpretante final.

\section{BIBLIOGRAFÍA}

AAMODT, Agnar AND NYGÅRD, Mads. "Different roles and mutual dependencies of data, information, and knowledge-an AI perspective on their integration", Data \& Knowledge Engineering, 1995, 16/ 3, 191-222.

ACKOFF, Russell L. "From data to wisdom", Journal of applied systems analysis, 1989, 16, 3-9.

BELL, Daniel: El advenimiento de la sociedad post-industrial: un intento de prognosis social.Madrid: Alianza, 1976, 378 págs.

BEYNON-DAVIES, Paul. "Neolithic informatics: The nature of information", International Journal of Information Management, 2009, 29/ 1, 3-14.

BODDY, David, BOONSTRA, Albert AND KENNEDY, Graham: Managing information systems: an organisational perspective.Essex: Pearson Education, 2005, 282 págs.

BOISOT, Max AND CANALS, Agustí. "Data, information and knowledge: have we got it right?", Journal of Evolutionary Economics, 2004, 14/ 1, 43-67.

BONFANTINI, Massimo A. "Sobre la connotación", Estudios de lingüística, 1984, 4, 149-158.

CASTAÑARES, Wenceslao: El signo: problemas semióticos y filosóficos. Tesis (PhD). Universidad Autónoma de Madrid, Departamento de Lógica, 1985, 457 págs.

---. La orientación semiótica. En: De la interpretación a la lectura. Madrid: Iberediciones, 1994, pp. 116-165. 
CHANDRASEKARAN, Balakrishnan, JOHNSON, Todd AND SMITH, Jack W. "Task structure analysis for knowledge modeling", Communications of the ACM, 1992, 33/ 9, 124-136.

CHIANG, Roger H. L., BARRON, Terence M. AND STOREY, Veda C. "Data, knowledge \& information in database and knowledge-based systems", Journal of Database Administration, 1992, 3/ 3, 12-20.

$\mathrm{CHOO}$, Chun Wei. "The knowing organization: How organizations use information to construct meaning, create knowledge and make decisions", International Journal of Information Management, 1996, 16/ 5, 329-340.

CODINA, Lluís. "El futuro de los estudios de Biblioteconomía y Documentación y su adecuación a la demanda social", Documentación de las Ciencias de la Información, 2002, 25, 217-234.

DAVENPORT, Thomas Hayes AND PRUSAK, Laurence: Working knowledge: how organizations manage what they know.Boston: Harvard Business School Press, 1998, 199 págs.

DRUCKER, Peter Ferdinand: La sociedad postcapitalista.Madrid: Apóstrofe, 1993, 224 págs.

ECO, Umberto: Signo.Barcelona: Labor, 1988, 216 págs.

GADOMSKI, Adam Maria. Meta-ontologival assumptions: information, preferences and knowledge universal interrelations (cognitive IPK architecture) [en línea]. Meta-Knowledge Engineering \& Management Server(trad.), [ref. de 10 de julio de 2014], 1999, Disponible en Web: http://erg4146.casaccia.enea.it/wwwerg26701/gad-dict.htm.

HEY, Jonathan (2004). The data, information, knowledge, wisdom chain: The metaphorical link. UNESCO.

LOW, David William. Data, information, knowledge: a semiotic-system's view for database design. In Proceedings of the Proceedings of the 53rd Annual Meeting of the ISSS-2009, Brisbane, Australia, 2009, May, 1-16.

MAGARIÑOS, Juan: La semiótica de los bordes: apuntes de metodología semiótica.Córdoba: Comunicarte, 2008, 439 págs.

MALHOTRA, Yogesh. "Tooks@work: Deciphering the knowledge management hype", The Journal for Quality and Participation, 1998, 21/ 4, 58-60.

MORRIS, Charles William: Signos, lenguaje y conducta.Buenos Aires: Losada, 1962, 341 págs.

NAKE, Frieder. Data, information, and knowledge. En: Kecheng Liu, Rodney J. Clarke, Peter Bøgh Andersen, Ronald K. Stamper AND El-Sayed Abou-Zeid eds. Organizational Semiotics: Evolving a Science of Information Systems IFIP TC8 / WG8.1 Working Conference on Organizational Semiotics: Evolving a Science of Information Systems July 23-25, 2001, Montreal, Quebec, Canada. New York: Springer, vol. 94, 2002, pp. 41-50.

NEWELL, Allen. "The knowledge level", Artificial Intelligence, 1982, 18/ 1, 87-127. 
NITECKI, Joseph Z. "The concept of information-knowledge continuum: implications for librarianship", Journal of Library history, 1985, 20/ 4, 387-407.

NONAKA, Ikujiro. "A dynamic theory of organizational knowledge creation", Organization Science, 1994, 5/ 1, 14-37.

PEIRCE, Charles Sanders. De una nueva lista de categorías [en línea]. Grupo de Estudios Peirceanos. Pilar Castrillo (trad.). Navarra, [ref. de 10 de julio de 2015], 1867 , Disponible en

Web: http://www.unav.es/gep/Categorias/NuevaListaCategorias.html.

---. Fundamento, objeto e interpretante [en línea]. Grupo de Estudios Peirceanos. Mariluz Restrepo (trad.). Navarra, [ref. de 10 de julio de 2015], 1897, Disponible en Web: http://www.unav.es/gep/FundamentoObjetoInterpretante.html.

---. La lógica considerada como semiótica [en línea]. Grupo de Estudios Peirceanos. Sara Barrena (trad.). Navarra, [ref. de 10 de julio de 2015], 1902, Disponible en Web: http://www.unav.es/gep/L75.pdf.

---. Nomenclatura y divisiones de las relaciones triádicas, hasta dónde están determinadas [en línea]. Grupo de Estudios Peirceanos. María Fernanda Benitti (trad.). Navarra, [ref. de 20 de julio de 2015], 1903, Disponible en Web: http://www.unav.es/gep/RelacionesTriadicas.html.

---. ¿Qué es el Pragmatismo? [en línea]. Grupo de Estudios Peirceanos. Norman Ahumada (trad.). Navarra, [ref. de 20 de julio de 2015], 1905, Disponible en Web: http://www.unav.es/gep/WhatPragmatismIs.html.

POLANY, Michael: Personal knowledge: towards a post-critical philosophy.London: Routledge \& Kegan Paul, 1962, 428 págs.

---: The tacit dimension.London: Routledge \& Kegan Paul, 1966, 108 págs.

POLLOCK, Neal (2002). Knowledge management and information technology (Know-IT Encyclopedia). Fort Belvoir: Defense Acquisition University Press, 384 págs.

PRADA MADRID, Ennio. "Los insumos invisibles de decisión: datos, información y conocimiento", Anales de Documentación, 2008, 11, 183-196.

REDONDO, Ignacio: El signo como medio: claves del pensamiento de C. S. Peirce para una teoría constitutiva de la comunicación. Universidad de Navarra, Departamento de Comunicación Pública, 2009, 411 págs.

ROS GARCÍA, María Del Rosario. "El gestor de la información, el gestor de marketing y el gestor del conocimiento", Documentación de las Ciencias de la Información, 2006, 29, 279-299.

ROWLEY, Jennifer. "The wisdom hierarchy: representations of the DIKW hierarchy", Journal of Information Science, 2007, 33/ 2, 163-180.

SCHREIBER, Guus, et al.: Knowledge engineering and management: The CommonKADS methodology. Cambridge: The MIT Press, 1999, 466 págs.

SHANNON, Claude Elwood AND WEAVER, Warren: The Mathematical Theory of Communication.Urbana: University of Illinois Press, 1971, 144 págs. 
SIMON, Herbert A. "Theories of bounded rationality", Decision and organization, 1972, 1/ 1, 161-176.

STAMPER, Ronald. Signs, information, norms and systems. En: Berit Holmqvist, Peter Bøgh Andersen, Heinz Klein AND Roland Posner eds. Signs of work: semiosis and information processing in organisations. New York: Walter de Gruyter, 1996, pp. 349-398.

STAMPER, Ronald, et al. "Understanding the roles of signs and norms in organizations-a semiotic approach to information systems design", Behaviour \& Information Technology, 2000, 19/ 1, 15-27.

SVEIBY, Karl Erik: Capital Intelectual: la Nueva riqueza de las empresas: Cómo medir y gestionar los activos intangibles para crear valor.Barcelona: Gestión 2000, 2000, 325 págs.

TUOMI, Ilkka. "Data is more than knowledge", Journal of Management Information Systems, 1999, 16/ 3, 107-121.

URIARTE, Filemón A.: Introduction to knowledge management.Jakarta: ASEAN Foundation, 2008, 168 págs.

ZELENY, Milan. "From knowledge to wisdom: on being informed and knowledgeable, becoming wise and ethical", International Journal of Information Technology \& Decision Making, 2006, 5/ 4, 751-762.

ZINS, Chaim. "Conceptual approaches for defining data, information, and knowledge", Journal of the American Society for Information Science and Technology, 2007, 58/ 4, 479-493. 